\title{
Atoms
}

Howard Bauchner, Editor-in-Chief

\section{The cost of Palivizumab}

As countries, even the US, discuss cost and benefit in healthcare, the debate continues about who should receive palivizumab and for how long. At a cost of roughly $\$ 1000$ per injection in the US, final decisions about who should receive the drug have substantial implications for the cost of child health. Since intensive care unit admission, assisted respiratory support and death are uncommon outcomes for premature infants who develop respiratory syncytial virus infection, the primary outcome in most studies has been hospitalisation rates. Unfortunately, variation in hospitalisation rates for premature infants who develop RSV is substantial, making any cost-effectiveness analysis fragile. In an interesting article from the Netherlands, Rietveld and colleagues add a new twist to the discussion. Recognising that the risk of infection varies substantially even over the winter months, they have created models that account not only for child characteristics, but also specific month of the year, for example, November versus January versus March. Perhaps it would be best to expand coverage to more high-risk infants, but reduce the number of months of coverage. See page 493

\section{More on UTIS}

The old adage of imaging following a diagnosis of UTI, and possible antibiotic prophylaxis is changing. NICE has released restrictive recommendations about which children with UTI should undergo imaging. Results from a number of recent studies suggest that prophylaxis may not prevent the development of recurrent UTIs or new renal scars, and may increase the risk of resistance bacteria. Dai et al, add to this continued discussion. In a systematic review of 11 studies that included 2046 patients they conclude that "this pooled analysis does not confirm that prophylactic antibiotics reduce the incidence of recurrent UTI in children." However, they add a cautionary comment, "However, the efficacy of long-term antibiotics for reducing recurrent symptomatic UTI cannot be disproven..." See page 499

\section{A guideline to assist clinicians in diagnosing brain tumours}

This paper was hotly debated at our Hanging Auction - our biweekly meeting where we discuss and debate which original research papers will be accepted for publication. The discussion centred on a number of issues: first, is this really a guideline; second, does any guideline which lacks high level evidence and largely reflects clinical wisdom worth producing; and third, are guidelines this detailed helpful for clinicians. Guidelines, like many other popular trends in medicine, seem to be past their prime years. Greater acknowledgment about the complexity of developing and updating guidelines, particularly when data are lacking, the need to account for both intellectual and financial conflict of interest and patient preference, has tempered the guideline movement. Even NICE in their more recent statements, appear to be downplaying the role of evidence in their recommendations - or at least how they report the level of evidence for each of their recommendations. At a recent scientist symposium in Canada which I helped organise, there was a wide ranging discussion about new ways to assimilate, report, and make recommendations (guidelines). I found the presentation by Jan Brozek, a member of the McMaster Group who has worked on the GRADE recommendations most enlightening. He made it quite clear that even if the evidence for a recommendation is high quality, but there is concern about patient acceptance, the final recommendation will not be enthusiastic. Back to this paper on detecting brain tumours in children I would love to hear from our readers if they think it is helpful and we have created an on-line poll to solicit your opinion. See page 534

\section{More on genetics}

Now that the time is approaching when every human being can have their entire genome scanned and entered into his or her medical record at a reasonable cost, debate has begun about whether this will change the face of medicine as we know it. Both Lancet and NEJM are approaching this question through case studies. In the Lancet piece ${ }^{1}$, investigators scanned the entire genome of a single man and discuss ways in which it might help him and his physicians make decisions about care. The NEJM $M^{2}$ series has just begun, but has taken a similar approach, although it appears more theoretical. The brave new world of personalised health appears around the corner - I continue to wonder if it will really make us a healthier society. I am sure it will help some individuals, although it may exacerbate healthcare disparities, but the great gains for society lay in changing adverse health behaviours, like curbing the obesity epidemic, reducing smoking, and encouraging individuals to exercise.

\section{Ketogenic diet}

I have always been sceptical about the effectiveness of the ketogenic diet to control seizures in children. Most of the early studies involved small numbers of patients, they were observational cohort studies, or case-series, and a Cochrane review in 2004 concluded that there was no reliable evidence to suggest that the diet was effective. Since then, a number of additional studies have been published. An excellent review by J H Cross and colleagues summarizes the recent literature - which suggests that the diet is effective - and also provides guidance around implementation of such a diet.

\section{See page 550}

\section{REFERENCES}

1. Ashley EA, Butte AJ, Wheeler MT, et al. Clinical assessment incorporating a personal genome. Lancet 2010;375:1525-35.

2. Feero GW, Guttmacher AE, Collins FS. Genomic medicine - an update primer. N Eng J Med 2010;362:2001-11. 